\title{
Paley type partial difference sets in non $p$-groups
}

\author{
John Polhill \\ Department of Mathematics, Computer Science, and Statistics \\ Bloomsburg University \\ Bloomsburg, PA 17815 \\ email : jpolhill@bloomu.edu
}

\begin{abstract}
By modifying a construction for Hadamard (Menon) difference sets we construct two infinite families of negative Latin square type partial difference sets in groups of the form $\mathbb{Z}_{3}{ }^{2} \times \mathbb{Z}_{p}{ }^{4 t}$ where $p$ is any odd prime. One of these families has the wellknown Paley parameters, which had previously only been constructed in $p$-groups. This provides new constructions of Hadamard matrices and implies the existence of many new strongly regular graphs including some that are conference graphs. As a corollary, we are able to construct Paley-Hadamard difference sets of the StantonSprott family in groups of the form $\mathbb{Z}_{3}^{2} \times \mathbb{Z}_{p}{ }^{4 t} \times \mathbb{Z}_{9 p^{4 t} \pm 2}$ when $9 p^{4 t} \pm 2$ is a prime power. These are new parameters for such difference sets.
\end{abstract}

\section{Introduction}

Let $G$ be a finite group of order $v$, and let $D$ be a subset of $G$ with cardinality $k$. Then $D$ is a $(v, k, \lambda)$-difference set (DS) if the list of differences $d_{1} d_{2}^{-1}, d_{1}, d_{2} \in D$ represents every nonidentity element in $G$ exactly $\lambda$ times. A difference set, $D$, is called reversible if $d \in D$ implies $d^{-1} \in D$. Hadamard (Menon) difference sets, having parameters $\left(4 u^{2}, 2 u^{2}-\right.$ $\left.u, u^{2}-u\right)$, are of particular interest due to the fact that their \pm 1 incidence matrices form Hadamard matrices. The text of Beth, Jungnickel, and Lenz [1] and the survey of Jungnickel [7] are excellent references for DSs.

Similarly, suppose $G$ is a finite group of order $v$ with a subset $D$ of order $k$ such that the differences $d_{1} d_{2}{ }^{-1}$ for $d_{1}, d_{2} \in D, d_{1} \neq d_{2}$ represent each nonidentity element of $D$ exactly $\lambda$ times and the nonidentity element of $G-D$ exactly $\mu$ times. Then $D$ is called a $(v, k, \lambda, \mu)$-partial difference set $(P D S)$ in $G$. When the identity $e \notin D$ and $D^{(-1)}=D$ we call the PDS $D$ regular. The survey article of Ma is a very good survey on PDSs [9]. A partial difference set having parameters $\left(n^{2}, r(n+1),-n+r^{2}+3 r, r^{2}+r\right)$ is called a negative Latin square type PDS.

Another important family of difference sets is the Paley-Hadamard difference sets having parameters $\left(v, \frac{v-1}{2}, \frac{v-3}{4}\right)$. When such a difference set also has the property that $G$ is the disjoint union of $D$, the inverses of the elements of $D$, and 0 , it is called a skew Hadamard difference set. Closely related to Paley-Hadamard difference sets are Paley type partial difference sets, having parameters $\left(v, \frac{v-1}{2}, \frac{v-5}{4}, \frac{v-1}{4}\right)$. Paley originally discovered 
these sets [10] along with the Paley-Hadamard difference sets in the context of Hadamard matrices, and in fact both Paley PDSs and Paley-Hadamard difference sets can be used to construct both Hadamard designs and matrices. In the survey of Ma [9] were the following questions:

"Questions 13.4. Suppose $G$ is an abelian group of order $v \equiv 1(\bmod 4)$. If $v$ is not a prime power, does there exist a Paley PDS in $G$ ? If $v$ is a prime power, does $G$ need to be elementary abelian?"

The articles of Davis as well as Leung and Ma give various constructions of Paley partial difference sets in $p$-groups ([3], [8]) that are not elementary abelian and thus answer the second question. In this paper, we will give the first construction of Paley PDSs in groups having an order which is not a prime power and therefore provide the first positive answer to the first of the questions. Using these Paley PDSs, we will also construct Paley-Hadamard DSs with new parameters.

There have also been relatively few constructions of negative Latin square type partial difference sets. Originally most of these were in nonelementary abelian groups, [9]. Recently, there have been constructions given in nonelementary abelian $p$-groups such as Davis and Xiang in [5] and Polhill [11]. Jørgensen and Klin [6] constructed negative Latin square type PDSs in groups of order 100. In this paper, we will not only construct the Paley PDSs but another pair of families of negative Latin square type PDSs in non $p$-groups as well.

Regular partial difference sets generate Cayley graphs which are strongly regular, and in particular the Paley-type and negative Latin square type PDSs give rise to conference graphs and negative Latin square type strongly regular graphs respectively.

Often DSs and PDSs are studied within the context of the group $\operatorname{ring} \mathbf{Z}[\mathbf{G}]$. For a subset $D$ in $G$ we can write $D=\sum_{d \in D} d$ and $D^{(-1)}=\sum_{d \in D} d^{-1}$. This is abuse of notation which is widely accepted; so that depending on the context $D$ will represent the difference set $D$ or the element $\sum_{d \in D} d$ in the group ring $\mathbb{Z}[G]$. Character theory is frequently used to simplify calculations with difference sets and partial difference sets in abelian groups. Turyn [13] and separately Yamamoto [18] first used character theory to study abelian difference sets. A character on an abelian group $G$ is a homomorphism from the group to the complex numbers with modulus 1 . The principal character is 1 on all the elements of $G$; any other character is called nonprincipal. One can naturally extend a character on $G$ to a homomorphism of the group ring $\mathbb{Z}[G]$ as follows: if $\chi$ is a character on $G$, then for an element $A=\sum_{g \in G} a_{g} g$ let $\chi(A)=\sum_{g \in G} a_{g} \chi(g)$ so that if $S$ is a subset of $G$, then $\chi(S)=\sum_{s \in S} \chi(s)$. See [13] for a proof of similar results to the following.

Theorem 1.1 (a) Let $G$ be an abelian group of order $v$ with a subset $D$ of cardinality $k$ and let $\lambda$ be a positive integer satisfying $\lambda(v-1)=k(k-1)$. Then $D$ is $a(v, k, \lambda)$-difference set in $G$ if and only if for every nonprincipal character $\chi$ on $G,|\chi(D)|=\sqrt{k-\lambda}$.

(b) Let $G$ be an abelian group of order $v$ with a subset $P$ of cardinality $k$ such that $0 \notin P$ with $k^{2}=k+\lambda k+\mu(v-k-1)$ for positive integers $\lambda$ and $\mu$. Then $P$ is a $(v, k, \lambda, \mu)$-partial difference set in $G$ if and only if for every nonprincipal character $\chi$ on $G, \chi(P)=\frac{(\lambda-\mu) \pm \sqrt{(\lambda-\mu)^{2}+4(k-\mu)}}{2}$. 


\section{Known Constructions of Hadamard Difference Sets}

Of particular relevance to this paper is the Hadamard difference set construction for $K \times$ $\mathbb{Z}_{p}{ }^{4 t}$ where $|K|=4$ and $p$ is an odd prime. Xia first constructed such difference sets for $p \equiv 3 \bmod 4$ in [16], and a simplified construction was given by Xiang and Chen [17] using the additive characters of finite fields. Then Wilson and Xiang [15] related the existence of this type of difference sets to certain projective sets, and finally Chen used their work to construct Hadamard difference sets in all groups $K \times \mathbb{Z}_{p}{ }^{4 t}$ for $|K|=4$ and $p$ an odd prime $[2]$.

Let $P G(k-1, q)$ denote the projective space of dimension $k-1$ over $F_{q}$ for $q$ a power of a prime; the corresponding vector space $V(k, q)$ for $P G(k-1, q)$ will have dimension $k$ over $F_{q}$. The elements of $P G(k-1, q)$ are the subspaces of $V(k, q)$, and, in particular, a projective point is a 1-dimensional subspace, a projective line is a 2-dimensional space, and a hyperplane is a $(k-1)$-dimensional subspace. A projective $\left(n, k, h_{1}, h_{2}\right)$ set $\mathcal{O}$ is a proper subset of the projective space $P G(k-1, q)$ with $n$ points $(n \neq 0)$ so that $\mathcal{O}$ intersects every hyperplane in $h_{1}$ or $h_{2}$ points. If we have that $\mathcal{O}=\left\{\left\langle y_{1}\right\rangle,\left\langle y_{2}\right\rangle, \ldots,\left\langle y_{n}\right\rangle\right\}$ then let $\Omega=\{x \in V(k, q) \mid\langle x\rangle \in \mathcal{O}\}$. The following lemma shows that $\mathcal{O}$ is a projective set if and only if $\Omega$ is a PDS in the additive group of the corresponding vector space under certain conditions. Wilson and Xiang have this result in [15].

Lemma 2.1 $\mathcal{O}$ is a projective $\left(n, k, h_{1}, h_{2}\right)$ set if and only if $\chi(\Omega)=q h_{1}-n$ or $q h_{2}-n$ for every nonprincipal additive character.

Using this lemma we see that $\mathcal{O}$ is a projective $\left(n, k, h_{1}, h_{2}\right)$ set if and only if $\Omega$ is a PDS in the additive group of $V(k, q)$ provided that $K^{2}=K+\lambda K+\mu(v-K-1)$ ( $K$ is the cardinality of $\Omega$, while $\lambda$ and $\mu$ are the usual PDS parameters).

Now consider the projective space $\Sigma_{3}=P G(3, q)$ for an odd prime power $q=p^{t}$. The additive group of the corresponding vector space is $\mathbb{Z}_{p}{ }^{4 t}$. Define a spread of $\Sigma_{3}$ to be a set of $q^{2}+1$ projective lines which are pairwise disjoint and partition the points of $\Sigma_{3}$. Finally, a subset of Type $Q$ is a projective $\left(\frac{q^{4}-1}{4(q-1)}, 4, \frac{(q-1)^{2}}{4}, \frac{(q+1)^{2}}{4}\right)$ set in $\Sigma_{3}$.

Theorem 2.2 (Xiang-Wilson [15]) Suppose that $S=\left\{L_{1}, L_{2}, \ldots, L_{q^{2}+1}\right\}$ is a spread of $\Sigma_{3}$. If there exist two subsets of Type $Q, C_{0}$ and $C_{1}$, in $\Sigma_{3}$ with the property that $\left|C_{0} \cap L_{i}\right|=(q+1) / 2$ for $1 \leq i \leq \frac{q^{2}+1}{2}$ and $\left|C_{1} \cap L_{j}\right|=(q+1) / 2$ for $\frac{q^{2}+1}{2}+1 \leq j \leq q^{2}+1$, then there exists a Hadamard difference set in $K \times \mathbb{Z}_{p}{ }^{4 t}$ for $|K|=4$.

We make some observations regarding this Theorem. If two such subsets of Type $\mathrm{Q}$ exist, then the sets

$$
\begin{gathered}
C_{2}=\cup_{1 \leq i \leq \frac{q^{2}+1}{2}} L_{i} \backslash C_{0} \text { and } \\
C_{3}=\cup_{\frac{q^{2}+1}{2}+1 \leq j \leq q^{2}+1} L_{j} \backslash C_{1}
\end{gathered}
$$

are also subsets of Type Q.

Now let $W=V(k, q)$ be the underlying vector space of $\Sigma_{3}$. Then let $\mathcal{C}_{i}=\{w \in$ $\left.W \mid\langle w\rangle \in C_{i}\right\}$ and $\mathcal{L}_{i}=\left\{w \in W \mid\langle w\rangle \in L_{i}\right\}$. 
Each of the four subsets $C_{i}$ in $\Sigma_{3}$ gives rise to a PDS $\mathcal{C}_{i}$ in $V(4, q)$ such that the character values of each are either $\frac{q^{2}-1}{4}$ or $\frac{q^{2}-1}{4}-q^{2}$ for nonprincipal additive characters on $V(4, q)$. The character sum will be $\frac{q^{2}-1}{4}-q^{2}$ for exactly one of the sets $\mathcal{C}_{0}, \mathcal{C}_{1}, \mathcal{C}_{2}, \mathcal{C}_{3}$ for any nonprincipal character.

To each of $\mathcal{C}_{0}$ and $\mathcal{C}_{2}$ we will add $\frac{q^{2}-1}{4}$ of the sets $\mathcal{L}_{j}$ which are disjoint from $\mathcal{C}_{0}$ and $\mathcal{C}_{2}$; we call this collection of $\frac{q^{2}-1}{4}$ projective lines $B_{1}$ with corresponding set $\mathcal{B}_{1}=\{w \in$ $\left.W \mid\langle w\rangle \in B_{1}\right\}$. The character sum on $\mathcal{B}_{1}$ will be either $-\frac{q^{2}-1}{4}$ or $q^{2}-\frac{q^{2}-1}{4}$, and when combined with the PDSs from subsets of Type Q, the character sums are either 0 or $\pm q^{2}$.

We similarly add $\frac{q^{2}-1}{4}$ of the sets $\mathcal{L}_{i}$ (disjoint from $\mathcal{C}_{1}$ and $\mathcal{C}_{3}$ ) to $\mathcal{C}_{1}$ and $\mathcal{C}_{3}$, and call this collection $B_{0}$. When we take the four sets

$$
D_{0}=\mathcal{C}_{0} \cup \mathcal{B}_{1}, \quad D_{1}=\mathcal{C}_{1} \cup \mathcal{B}_{0}, \quad D_{2}=\mathcal{C}_{2} \cup \mathcal{B}_{1}, \quad D_{3}=\mathcal{C}_{3} \cup \mathcal{B}_{0},
$$

the character sums will be such that for any nonprincipal character $\chi$ on $G=\mathbb{Z}_{p}{ }^{4 t}, \chi\left(D_{i}\right)=$ $\pm q^{2}$ for exactly one $i$ and $\chi\left(D_{k}\right)=0$ for all other $k$. Then the four sets $G \backslash D_{0}, D_{1}, D_{2}, D_{3}$ form a $\left(\frac{p^{4}-p^{2}}{2}, p^{2}, 4,+\right)$ covering extended building set using the terminology from [4], so that if $K=\left\{a_{0}, a_{1}, a_{2}, a_{3}\right\}$ then

$$
H=a_{0}\left(G \backslash D_{0}\right) \cup a_{1} D_{1} \cup a_{2} D_{2} \cup a_{3} D_{3}
$$

is a Hadamard difference set in $K \times \mathbb{Z}_{p}{ }^{4 t}$.

We form two more sets $B_{2}$ and $B_{3}$ as follows: $\mathcal{B}_{2}=\left(\mathcal{C}_{0} \cup \mathcal{C}_{2}\right) \backslash \mathcal{B}_{0}$ and $\mathcal{B}_{3}=\left(\mathcal{C}_{1} \cup \mathcal{C}_{3}\right) \backslash \mathcal{B}_{1}$ so that each of $B_{2}$ and $B_{3}$ are unions of $\frac{q^{2}+3}{4}$ projective lines. The properties of character sums on the $\mathcal{B}_{i}$ are given in the following lemma.

Lemma 2.3 Let $\chi$ be a nonprincipal character on $V(4, q)$. Then $\chi\left(\mathcal{B}_{0}\right), \chi\left(\mathcal{B}_{1}\right) \in\left\{-\frac{q^{2}-1}{4}, q^{2}-\right.$ $\left.\frac{q^{2}-1}{4}\right\}$ and $\chi\left(\mathcal{B}_{2}\right), \chi\left(\mathcal{B}_{3}\right) \in\left\{-\frac{q^{2}+3}{4}, q^{2}-\frac{q^{2}+3}{4}\right\}$. Furthermore, exactly one $\mathcal{B}_{i}$ will have a positive character sum.

Proof: The character sums immediately follow from Theorem 1.1. The fact that there will be exactly one positive character sum follows from the fact that $\Sigma_{3}=B_{0} \cup B_{1} \cup B_{2} \cup B_{3}$.

Now we wish to form two more sets $L$ and $M$ in the additive group of $V(4, q) . L=$ $\mathcal{B}_{1} \cup \mathcal{B}_{2}$. The set $L$, viewed projectively, is a union of $\frac{q^{2}+1}{2}$ projective lines, and in fact is a Paley-type PDS in the additive group of $V(4, q)$. The set $M=\mathcal{B}_{2} \cup \mathcal{B}_{3}$, viewed projectively, is a union of $\frac{q^{2}+3}{2}$ lines and is also a PDS in the additive group of $V(4, q)$. These PDSs have special properties with respect to the sets $D_{i}$ as given in the following key Lemmas.

Lemma 2.4 Let $\chi$ be a nonprincipal character on $V(4, q)$. For $i=0$ or 2 , if $\chi\left(G \backslash D_{i}\right)=$ $\pm q^{2}$ then $\chi(L)=\frac{\mp q^{2}-1}{2}$. For $i=1$ or 3 , if $\chi\left(D_{i}\right)= \pm q^{2}$ then $\chi(L)=\frac{\mp q^{2}-1}{2}$. 
Proof: There will be exactly one $\mathcal{L}_{i}$ on which $\chi$ is principal. Suppose $\chi\left(D_{0}\right)=q^{2}$ so $\chi\left(G \backslash D_{0}\right)=-q^{2}$. Then it must be the case that $\chi\left(\mathcal{B}_{1}\right)$ is positive. It follows that $\chi(L)=\frac{q^{2}-1}{2}$. If instead $\chi\left(D_{0}\right)=-q^{2}$ then $\chi\left(G \backslash D_{0}\right)=q^{2}$. It follows that $\chi\left(\mathcal{B}_{1}\right)$ is negative. Moreover, $\chi\left(\mathcal{C}_{0}\right)=\frac{q^{2}-1}{4}-q^{2}$ so that $\chi\left(\mathcal{C}_{0} \cup \mathcal{C}_{2}\right)=\chi\left(\mathcal{B}_{0} \cup \mathcal{B}_{2}\right)=\frac{-q^{2}-1}{2}$. This ensures that $\chi\left(\mathcal{B}_{0}\right)$ and $\chi\left(\mathcal{B}_{2}\right)$ must both be negative. Then it must be the case that $\chi\left(\mathcal{B}_{3}\right)$ is positive, so that $\chi(L)=\frac{-q^{2}-1}{2}$. The cases $i \neq 0$ are similar.

Lemma 2.5 Let $\chi$ be a nonprincipal character on $V(4, q)$. If $\chi\left(D_{i}\right)= \pm q^{2}$ then $\chi(M)=$ $\frac{\mp q^{2}-3}{2}$.

\section{$3 \quad$ Paley PDSs in Non $p$-Groups}

Let $G=\mathbb{Z}_{p}{ }^{4 t}$. Using the same sets $D_{i} \subset G$ as in Xiang-Wilson's Theorem above, we can replace the group $K$ with $\mathbb{Z}_{3}{ }^{2}$ and will be able to form negative Latin square type PDSs in any group of the form $G^{\prime}=\mathbb{Z}_{3}^{2} \times G$. Let $H_{0}, H_{1}, H_{2}$, and $H_{3}$ be the subgroups of order 3 in $\mathbb{Z}_{3}{ }^{2} . H_{i}{ }^{*}=H_{i} \backslash\{(0,0)\}$ so that $H_{i}{ }^{*} \cap H_{j}{ }^{*}=\emptyset$ for $i \neq j$. This will ensure that the set $D$ given in Theorem 3.1 is not a multiset.

Theorem 3.1 (Paley partial difference sets in non $p$-groups) Let $G^{\prime}=\mathbb{Z}_{3}^{2} \times G$ where $G=\mathbb{Z}_{p}{ }^{4 t}$ for an odd prime $p$. Then the set $D=(\{(0,0)\} \times L) \cup\left(H_{0}{ }^{*} \times\left(G \backslash D_{0}\right)\right) \cup$ $\left(H_{1}{ }^{*} \times D_{1}\right) \cup\left(H_{2}{ }^{*} \times\left(G \backslash D_{2}\right)\right) \cup\left(H_{3}{ }^{*} \times D_{3}\right)$ is a $\left(9 p^{4 t}, \frac{9 p^{4 t}-1}{2}, \frac{9 p^{4 t}-5}{4}, \frac{9 p^{4 t}-1}{4}\right)$ Paley-type $P D S$ in $G^{\prime}$.

Proof: First we check to see that $D$ has the correct cardinality.

$|D|=|L|+2\left|G \backslash D_{0}\right|+2\left|D_{1}\right|+2\left|G \backslash D_{2}\right|+2\left|D_{3}\right|=\frac{p^{4 t}-1}{2}+4|G|=\frac{p^{4 t}-1}{2}+4 p^{4 t}=\frac{\left|G^{\prime}\right|-1}{2}$.

Suppose that $\phi$ is a nonprincipal character on $G^{\prime}=\mathbb{Z}_{3}^{2} \times G$. Then $\phi=\chi \otimes \psi$, where $\chi$ is a character on $\mathbb{Z}_{3}^{2}$ and $\psi$ is a character on $G$. To use Theorem 1.1, we need to show that $\phi(D)=\frac{ \pm 3 p^{2 t}-1}{2}$.

Case 1: $\chi$ is principal on $\mathbb{Z}_{3}^{2}$, but $\psi$ is nonprincipal on $G$. Then $\chi\left(H_{i}^{*}\right)=2$ for all $i$. $\psi\left(D_{j}\right)= \pm q^{2}= \pm p^{2 t}$ for exactly one $j$, and $\psi\left(D_{i}\right)=0$ for $i \neq j$. If $j=1$ or 3 then by Lemma 2.4, if $\psi\left(D_{j}\right)=p^{2 t}$ then $\psi(L)=\frac{-p^{2 t}-1}{2}$ while if $\psi\left(D_{j}\right)=-p^{2 t}$ then $\psi(L)=\frac{p^{2 t}-1}{2}$. We will have then:

$$
\phi(D)=\frac{ \pm p^{2 t}-1}{2}+2\left(\mp p^{2 t}\right)=\frac{\mp 3 p^{2 t}-1}{2} .
$$

A similar argument works for $j=0$ or 2 .

Case 2: $\quad \chi$ is nonprincipal on $\mathbb{Z}_{3}^{2}$, but $\psi$ is principal on $G$. Then $\psi(L)=\frac{p^{4 t}-1}{2}$, $\psi\left(G \backslash D_{i}\right)=\left|G \backslash D_{i}\right|=\frac{p^{4 t}+p^{2 t}}{2}$, and $\psi\left(D_{i}\right)=\left|D_{i}\right|=\frac{p^{4 t}-p^{2 t}}{2}$. $\chi$ will be principal on exactly one of the $H_{j}$ so that $\chi\left(H_{j}^{*}\right)=2$ while $\chi\left(H_{i}^{*}\right)=-1$ for $i \neq j$. If $j=0$ or 2 we get:

$$
\chi(D)=|L|+(2-1)\left(\frac{p^{4 t}+p^{2 t}}{2}\right)+(-1-1)\left(\frac{p^{4 t}-p^{2 t}}{2}\right)=\frac{3 p^{2 t}-1}{2} .
$$


If $j=1$ or 3 we get:

$$
\phi(D)=|L|+(-1-1)\left(\frac{p^{4 t}+p^{2 t}}{2}\right)+(2-1)\left(\frac{p^{4 t}-p^{2 t}}{2}\right)=\frac{-3 p^{2 t}-1}{2} .
$$

Case 3: Suppose that both $\chi$ and $\psi$ are nonprincipal. Then $\chi$ will be principal on exactly one of the $H_{k}$ so that $\chi\left(H_{k}^{*}\right)=2$ while $\chi\left(H_{i}^{*}\right)=-1$ for $i \neq k . \psi\left(D_{j}\right)= \pm q^{2}= \pm p^{2 t}$ for exactly one $j$, and $\psi\left(D_{i}\right)=0$ for $i \neq j$. If $j=0$ or 2 then by Lemma 2.4, if $\psi\left(G \backslash D_{j}\right)=p^{2 t}$ then $\psi(L)=\frac{-p^{2 t}-1}{2}$ while if $\psi\left(G \backslash D_{j}\right)=-p^{2 t}$ then $\psi(L)=\frac{p^{2 t}-1}{2}$. If $k=j$ we get:

$$
\phi(D)=\frac{ \pm p^{2 t}-1}{2}+2\left(\mp p^{2 t}\right)=\frac{\mp 3 p^{2 t}-1}{2} .
$$

If $k \neq j$ then we get:

$$
\phi(D)=\frac{ \pm p^{2 t}-1}{2}-1\left(\mp p^{2 t}\right)=\frac{ \pm 3 p^{2 t}-1}{2} .
$$

A similar argument works for $j=1$ or 3 .

We have hence provided a method to construct Paley PDSs in certain groups having an order that is not a prime power. The following theorem gives another family of negative Latin square type PDSs that will generate a family of negative Latin square type graphs with number of vertices that is not a prime power. We omit the proof since it is extremely similar to the previous.

Theorem 3.2 Let $G^{\prime}=\mathbb{Z}_{3}^{2} \times G$ where $G=\mathbb{Z}_{p}{ }^{4 t}$ for an odd prime $p$. Then the set $S=(\{(0,0)\} \times M) \cup\left(H_{0}{ }^{*} \times D_{0}\right) \cup\left(H_{1}{ }^{*} \times D_{1}\right) \cup\left(H_{2}{ }^{*} \times D_{2}\right) \cup\left(H_{3}{ }^{*} \times D_{3}\right)$ is a $\left(9 p^{4 t}, r\left(3 p^{2 t}+\right.\right.$ $\left.1),-3 p^{2 t}+r^{2}+3 r, r^{2}+r\right)$-negative Latin square type PDS in $G^{\prime}$ for $r=\frac{3 p^{2 t}-3}{2}$.

We can also get another set of parameters for negative Latin square type partial difference sets by taking $T=\left(G^{\prime} \times G\right)^{*} \backslash S$. As an example, in the group $\mathbb{Z}_{3}{ }^{2} \times \mathbb{Z}_{5}{ }^{4}$ we obtain from Theorem 3.1 a $\left(75^{2}, 37(75+1),-75+37^{2}+3(37), 37^{2}+37\right)$-Paley PDS which we call $D$. From Theorem 3.2 we obtain PDSs $S$ and $T$ which are $\left(75^{2}, 36(75+1),-75+36^{2}+\right.$ $\left.3(36), 36^{2}+36\right)$ - and $\left(75^{2}, 38(75+1),-75+38^{2}+3(38), 38^{2}+38\right)$-negative Latin square type partial difference sets. Thus we have three strongly regular graphs $D, S$, and $T$ of the negative Latin square type on $75^{2}=5625$ vertices, having degrees 2812,2736 , and 2888 respectively. The Cayley graph from $D$ will in particular be a conference graph.

\section{New Paley-Hadamard Difference Sets}

We will now show how the Paley PDSs constructed in Theorem 3.1 can be used to construct new Paley-Hadamard difference sets. The following is due to Stanton and Sprott [12].

Theorem 4.1 Suppose that $q$ and $q+2$ are both prime powers. Then there is a $(q(q+$ $\left.2), \frac{q(q+2)-1}{2}, \frac{q(q+2)-3}{2}\right)$-Hadamard difference set in $E A(q) \times E A(q+2)$, where $E A(q)$ denotes the elementary abelian group or order $q$ and is the additive group of $F_{q}$. 
In [14], there are the following generalizations of Stanton and Sprott's earlier result.

Theorem 4.2 Suppose that the group $G$ has a $\left(v, \frac{v-1}{2}, \frac{v-5}{4}, \frac{v-1}{4}\right)$-Paley partial difference set $P$ with $0 \notin P$ and that the group $G^{\prime}$ having order $v+2$ has a $\left(v+2, \frac{v+1}{2}, \frac{v-1}{4}\right)$-skew Hadamard difference set $S$. Then $D=(G \times\{0\}) \cup(P \times S) \cup\left(\left(G^{*} \backslash P\right) \times\left(G^{*} \backslash S\right)\right)$ is a $\left(v(v+2), \frac{v(v+2)-1}{2}, \frac{v(v+2)-3}{4}\right)$ - Paley-Hadamard difference set in the group $G \times G^{\prime}$.

Theorem 4.3 Suppose that the group $G$ has a $\left(v, \frac{v-1}{2}, \frac{v-5}{4}, \frac{v-1}{4}\right)$-Paley partial difference set $P$ with $0 \notin P$ and that the group having order $v-2$ has a $\left(v-2, \frac{v-3}{2}, \frac{v-5}{4}\right)$-skew Hadamard difference set $S$. Then $D=\left(\{0\} \times G^{\prime}\right) \cup(P \times S) \cup\left(\left(G^{*} \backslash P\right) \times\left(G^{\prime *} \backslash S\right)\right)$ is a $\left(v(v-2), \frac{v(v-2)-1}{2}, \frac{v(v-2)-3}{4}\right)$ - Paley-Hadamard difference set in the group $G \times G^{\prime}$.

Combining Theorem 3.1 with these two results we can get new Stanton-Sprott difference sets. These difference sets will have parameters distinct from previous constructions. A few examples of such Paley-Hadamard DSs are in the following groups: $\mathbb{Z}_{3}^{2} \times \mathbb{Z}_{5}{ }^{4} \times \mathbb{Z}_{5623}$, $\mathbb{Z}_{3}{ }^{2} \times \mathbb{Z}_{5}{ }^{8} \times \mathbb{Z}_{3515623}, \mathbb{Z}_{3}^{2} \times \mathbb{Z}_{5}{ }^{12} \times \mathbb{Z}_{2197265627}, \mathbb{Z}_{3}^{2} \times \mathbb{Z}_{7}^{4} \times \mathbb{Z}_{21611}$, and $\mathbb{Z}_{3}{ }^{2} \times \mathbb{Z}_{7}{ }^{8} \times \mathbb{Z}_{51883211}$. In each case, we have a Paley PDS from Theorem 3.1 in the first two components and a skew Hadamard difference set in the latter component since the latter is $\mathbb{Z}_{p}$ for a prime $p \equiv 3(\bmod 4)$. We summarize with the following corollary.

Corollary 4.4 Suppose that for an odd prime $p$ that $9 p^{4 t} \pm 2$ is a prime power. Then there is a Paley-Hadamard difference set in $\mathbb{Z}_{3}^{2} \times \mathbb{Z}_{p}{ }^{4 t} \times \mathbb{Z}_{9 p^{4 t} \pm 2}$.

Acknowledgments: The author would like to thank Dr. Qing Xiang for his help with this paper. Funding was provided by the 2008-09 Bloomsburg University Research and Disciplinary Competition.

\section{References}

[1] T. Beth, D. Jungnickel, and H. Lenz. Design Theory, Second ed. Encyclopedia of Mathematics and its Applications, 78. Cambridge: Cambridge University Press, 1999.

[2] Y. Q. Chen, On the existence of abelian Hadamard difference sets and a new family of difference sets. Finite Fields and their Applications, 3, 234-256, 1997.

[3] J. A. Davis, Partial difference sets in p-groups. Arch. Math., 63, 103-110, 1994.

[4] J. A. Davis and J. Jedwab. A unifying construction for difference sets. J. Comb. Th. (A), 80 (1), 13-78, 1997.

[5] J. A. Davis and Q. Xiang, Amorphic association schemes with negative Latin squaretype graphs. Finite Fields Appl., 12, 595-612, 2006.

[6] L. K. Jørgensen and M. Klin, Switching of edges in strongly regular graphs. I. A family of partial difference sets on 100 vertices. Electron. J. Combin, 10, 2003. 
[7] D. Jungnickel, Difference Sets. Contemporary Design Theory, Wiley-Intersci. Ser. Discrete Math Optim., New York: Wiley, 1992. 241-324.

[8] K. H. Leung and S. L. Ma, Partial difference sets with Paley parameters. Bull. London Math. Soc., 27, 553-564, 1995.

[9] S. L. Ma, A Survey of partial difference sets. Designs, Codes, and Cryptography, 4, 221-261, 1994.

[10] R. E. A. C. Paley, On orthogonal matrices. J. Math and Phys., 12, 311-320, 1933.

[11] J. Polhill, New negative Latin square type partial difference sets in nonelementary abelian 2-groups and 3-groups. Designs, Codes, and Cryptography, 46 (3), 365-377, 2008.

[12] R. G. Stanton and D. A. Sprott, A family of difference sets. Canad. J. Math, 13, 73-77, 1958.

[13] R. J. Turyn, Character sums and difference sets. Pacific J. Math., 15, 319-346, 1965.

[14] G. Weng and L. Hu, Some results on skew Hadamard difference sets. Designs, Codes, and Cryptography, 50 (1), 93-105, 2009.

[15] R. M. Wilson and Q. Xiang, Constructions of Hadamard difference sets. J. Comb. Th. (A), 77, 148-160, 1997.

[16] M.-Y. Xia, Some infinite classes of special Williamson matrices and difference sets. $J$. Comb. Th. (A), 61, 230-242, 1992.

[17] Q. Xiang and Y. Q. Chen. On Xia's construction of Hadamard difference sets. Finite Fields and Their Applications, 2, 87-95, 1996.

[18] K. Yamamoto. Decomposition fields of difference sets. Pacific J. Math., 13: 337-352, 1963. 